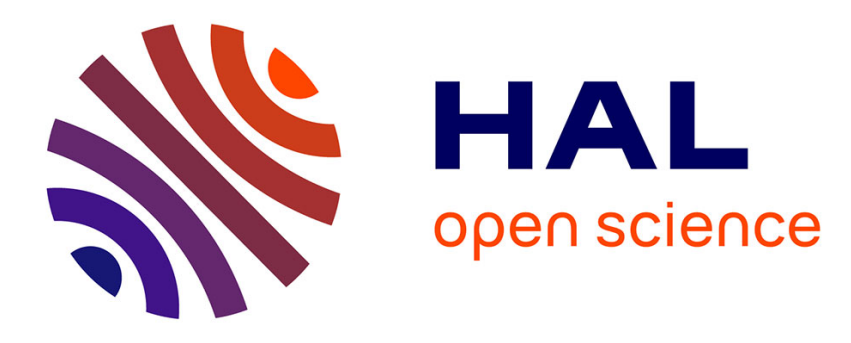

\title{
La description du français calédonien : état des lieux
}

Christine Pauleau

\section{To cite this version:}

Christine Pauleau. La description du français calédonien : état des lieux. Langages, 2016, 203, pp.2136. hal-01987735

\section{HAL Id: hal-01987735 \\ https://hal.parisnanterre.fr/hal-01987735}

Submitted on 21 Jan 2019

HAL is a multi-disciplinary open access archive for the deposit and dissemination of scientific research documents, whether they are published or not. The documents may come from teaching and research institutions in France or abroad, or from public or private research centers.
L'archive ouverte pluridisciplinaire HAL, est destinée au dépôt et à la diffusion de documents scientifiques de niveau recherche, publiés ou non, émanant des établissements d'enseignement et de recherche français ou étrangers, des laboratoires publics ou privés. 


\section{La description du français calédonien : état des lieux}

\section{INTRODUCTION}

L'Atlas de la Nouvelle-Calédonie (IRD 2012), au chapitre "Les populations " consacre un sous-chapitre aux langues kanak (op. cit. : 119-123). Mais les autres langues en usage en Nouvelle-Calédonie, pourtant nombreuses, ne font pas l'objet d'un autre sous-chapitre : faute de place, les coordinateurs ont dû, à regrets, faire un choix entre les thèmes linguistiques à développer ${ }^{1}$ et il était, bien sûr, plus que légitime que ce choix revienne aux langues kanak en tant que langues autochtones. Les "autres langues" sont néanmoins évoquées (op. cit. : 119), " liées à l'histoire de la colonisation (français, javanais, vietnamien, etc.) ou à des migrations récentes (tahitien, wallisien, futunien) ». Sont mentionnés également

[les] regroupements de population kanak autour de la mission de Saint-Louis, près de Nouméa, au cours de la seconde moitié du XIX $X^{\mathrm{e}}$ siècle [qui] sont à l'origine de l'émergence d'un créole à base lexicale française, le tayo. (IRD, 2012 : 119)

Pour en venir au français calédonien, forme prise par la langue française en Nouvelle-Calédonie, qui fait l'objet de notre propos ici, celui-ci n'est présent dans l'Atlas que par le lexique qui se trouve en fin d'ouvrage : certains régionalismes locaux utilisés dans les diverses planches de cet atlas (termes du domaine de la flore et de la faune, ou mots de la langue générale) ont dû, en effet, être définis pour que le lecteur extérieur ne soit pas gêné. Ces faits anecdotiques révèlent le peu de cas qui est souvent fait, dans un espace géographique francophone multilingue, des formes régionales du français par rapport aux langues autochtones ou aux faits de contacts linguistiques, même dans des ouvrages de haute

1. Communication personnelle de 2013 avec un des coordinateurs scientifiques de l'ouvrage. 
qualité scientifique. Nous illustrerons, dans la suite de cet article, cet état de fait : d'une part, nous montrerons succinctement (§ 2) la vitalité des recherches menées sur les langues kanak de Nouvelle-Calédonie ou sur le créole ; d'autre part, nous ferons $(\S 3)$ un état des lieux des travaux, peu nombreux, portant sur le français calédonien pour mettre en évidence l'absence criante de description dans certains domaines linguistiques.

\section{DES TRAVAUX LINGUISTIQUES PORTANT SURTOUT SUR LES LANGUES KANAK ET LE CRÉOLE}

Les travaux linguistiques portant sur la Nouvelle-Calédonie sont consacrés surtout à la description des langues kanak, d'une part, du créole, d'autre part. Nous évoquerons rapidement ici ces travaux pour situer les études sur le français calédonien par rapport à l'ensemble des travaux produits sur les langues en présence dans l'archipel calédonien ${ }^{2}$.

La description des langues kanak porte sur les domaines du lexique, de la syntaxe, de la morphologie, de la phonologie, de la sociolinguistique des langues en contact ou encore sur des domaines littéraires abordant la question de la tradition orale $^{3}$. Ce sont principalement des linguistes francophones de l'Hexagone ou de Nouvelle-Calédonie (ou anglophones d'Australie et de Nouvelle-Zélande) qui publient sur les langues kanak. Le bel Atlas de la Nouvelle-Calédonie (IRD 2012), dans la planche linguistique consacrée aux langues kanak, donne les « orientations bibliographiques » suivantes :

Outre la dizaine d'ouvrages (dictionnaires et études grammaticales de plusieurs langues kanak) publiés dans la collection «Langues et culture du Pacifique » des éditions Peeters, citons :

Cerquiglini B. (dir.), 2003 -Les langues de France, Paris, PUF : 347-435.

Haudricourt A.-G., Ozanne-Rivierre F., 1982 -Dictionnaire thématique des langues de la région de Hienghène (Nouvelle-Calédonie), Selaf-Peeters, Lacito-documents 4.

Lynch J., Ross M., Crowley T., 2002 -The Oceanic Languages, Curzon Press.

Ross M., Pawley A., Osmond M., 1998, 2003, 2008, 2011 -The lexicon of Proto

Oceanic. The Culture and Environment of Ancestral Oceanic Society, Canberra, Pacific Linguistics [...]. (IRD, 2012 : 122)

La description du créole de Nouvelle-Calédonie (le tayo) porte sur les mêmes domaines que ceux cités supra pour les langues kanak avec, en particulier, les publications multiples du linguiste néo-zélandais $C$. Corne sur le sujet dans les années 90, celles du Journal of Pidgin and Creole Languages (Ohio State University),

2. Rappelons également que l'article de Ehrhart (2016, ce volume) est consacré au créole et aux langues kanak dans leur relation écologique avec le français calédonien.

3. Voici les liens vers les principaux sites Internet sur lesquels on trouve des bibliographies concernant ces domaines : le site gouvernemental de l'Académie des Langues Kanak (ALK) [http://www.alk.gouv.nc/portal/ page/portal/alk/langues, consulté le 29-06-2016] et le site Océanie du Laboratoire des Langues et Civilisations à Tradition Orales (LACITU-CNRS) [http://lacito.vjt.cnrs.tr/ALC/oceanie.htm, consulté le 29-06-2016]. 
ainsi que les ouvrages de S. Ehrhart, spécialiste du tayo depuis presque trente ans (nous renvoyons pour toute autre précision à son article dans le présent ouvrage) ${ }^{4}$.

\section{3. ÉTAT DE LA RECHERCHE SUR LE FRANÇAIS CALÉDONIEN ${ }^{5}$}

Pour sa part, le français calédonien est étudié depuis une époque récente et de façon beaucoup moins soutenue que ne le sont notamment les langues kanak. En effet, l'isolement géographique de la Nouvelle-Calédonie par rapport à l'Occident a bien évidemment des répercussions sur la visibilité de la culture de ce pays et, si la culture kanak a bénéficié de l'enthousiasme pour les langues et cultures " orientales", ce n'est pas le cas de la culture franco-calédonienne. Cette dernière est le produit d'un métissage entre « occident » et " orient » resté méconnu, tout comme sa littérature, sa langue et les travaux de recherche portant sur ces champs culturels.

Nous verrons que seul le domaine du lexique du français calédonien fait véritablement l'objet de recherches, en outre quasi exclusivement de manière lexicographique. Quelques travaux ont certes été menés sur le plan phonétique ou sur des thèmes sociolinguistiques mais de manière ponctuelle et certains domaines, tels que celui de la syntaxe, sont encore vierges.

Il s'agit dans les paragraphes qui suivent d'effectuer un état des lieux des travaux existants et de rendre compte de ces recherches pour ainsi décrire les étapes d'un travail méconnu effectué sur un des terrains les plus isolés de la francophonie, terrain ignoré de la plupart des linguistes.

\subsection{Travaux concernant le lexique du français calédonien}

\subsubsection{L'Observatoire du français dans le Pacifique}

Les seuls travaux en sciences du langage menés sur ce terrain ont été, jusque dans les années 90, ceux de chercheurs néo-zélandais. D'une grande qualité scientifique, ces recherches, menées principalement dans le domaine du lexique, ont été non seulement une source d'inspiration fondatrice mais aussi une indispensable base méthodologique et documentaire pour entreprendre les premières descriptions lexicographiques du français calédonien (Pauleau 1995a).

\footnotetext{
4. Mentionnons également l'excellent ouvrage d'anthropologie du langage de Barnèche (2005), qui traite de l'usage et de la représentation des langues, y compris du français et du tayo, dans les milieux populaires océaniens de Nouméa.

5. Il est bien entendu que seuls les travaux scientifiques sont présentés ici. Ne sont pas évoqués les lexiques divers (certes peu nombreux comparés à d'autres terrains) tels que celui du site Internet Croix du sud (depuis 2000) ou que l'ouvrage, beaucoup plus ancien, de la Fédération des Euvres Laïques (1983). Ces documents constituent, en revanche, des corpus précieux et sont largement consultés en tant que tels lors des observations de terrain (Pauleau, $2007: 28$ ).
} 
Les linguistes du département des Langues Romanes de l'Université d'Auckland (Nouvelle-Zélande), associés à l'Institut National de la Langue Française (InaLF), publièrent une revue intitulée Observatoire $d u$ français dans le Pacifique : études et documents pendant plus de dix ans (CNRS-INALF 1983-1996). K. J. Hollyman, son épouse, J. Glasgow, et C. Corne formaient une équipe de linguistes francisants, se rendant régulièrement dans l'archipel calédonien voisin, observant pendant des décennies les usages oraux et écrits des locuteurs francophones de Nouvelle-Calédonie, cela dès les années 50-60. J. Glasgow soutint en 1968 une thèse sur le vocabulaire de l'élevage calédonien, vocabulaire issu du lexique de l'élevage australien ${ }^{6}$. L'Observatoire publia des textes de description divers, notamment de description différentielle : des travaux divers de C. Corne, s'étendant au moins de 1989 à 1996, au précieux et vaste répertoire alphabétique lexical contenant les expressions jugées particulières par rapport à la norme de référence (il s'agit de la rubrique de l'Observatoire appelée " Datations et témoignages d'enquête »; voir en annexe une page de cette rubrique). Pour chaque lexie étaient notées une définition succincte, une attestation orale et/ou écrite datée (des années 50 aux années 90) accompagnée de précisions sur le locuteur, d'informations sur l'absence ou la présence de la lexie dans les dictionnaires de français «standard ». Ces chercheurs, adoptant un point de vue non prescriptif, qui sera celui de toutes les descriptions par la suite, ont construit de solides matériaux pour la recherche en lexicographique différentielle calédonienne. De fait, ils ont également posé l'hypothèse, par la récolte de ces matériaux, de l'existence de diatopismes francophones sur le Territoire d'Outre-Mer français qu'était à cette époque la Nouvelle-Calédonie (voir l'introduction du présent ouvrage (Pauleau 2016b) pour des précisions sur le statut actuel).

\subsubsection{La description lexicologique et lexicographique du français calédonien}

Sur la voie tracée par les linguistes néo-zélandais évoqués supra, le domaine lexical est celui qui a retenu quasiment toute l'attention des travaux portant sur le français calédonien (p. ex. Pauleau 1995, 1997, 2006, 2007). La première étape du travail a donné lieu à la publication d'un ouvrage lexicographique (Pauleau 1995a). Elle se proposait de démontrer et de cerner l'existence du français calédonien, sa vitalité, son extension, son usage, ses aspects sociolinguistiques, avec une approche polylectale ${ }^{7}$. L'observation, non prescriptive, de la diversité des usages (nous pourrions dire de la diversité " des français calédoniens " ${ }^{8}$ )

\footnotetext{
6. Les exemples lexicaux emblématiques sont, par exemple, le nom de stockman donné aux ouvriers agricoles rentrant le bétail à cheval (à la manière australienne) et celui de station donné à la ferme : le lexique agricole foisonne ainsi d'emprunts à l'anglais austral.

7. Selon les Principes de grammaire polylectale (Berrendonner, Le Guern \& Puech 1983) prenant en compte une multiplicité de sous-variétés internes à la variété de langue en question.

8. Voir à ce sujet la situation sociolinguistique du français en Nouvelle-Calédonie présentée en introduction du présent ouvrage (Pauleau 2016b).
} 
a, selon ce principe polylectal, été effectuée selon les groupes sociaux, ethnolinguistiques, générationnels, culturels, présents dans l'archipel calédonien. Des enquêtes de terrain ont été menées sur la base d'un échantillon représentatif de la population : une soixantaine de locuteurs répartis dans les divers groupes cités précédemment, à Nouméa, ville abritant des citadins, mais aussi des ruraux ou ex-ruraux, où cohabitent tous les groupes socioculturels. Observation directe et indirecte, entretiens directifs et non-directifs, enregistrements, prises de notes, questionnaires écrits et oraux, multiples corpus écrits (littérature, presse, écrits divers publiques ou privés) et oraux (oral publique des médias - radio, télévision -, oral privé de conversation); ces enquêtes ont été fondamentales pour tendre vers l'objectivité descriptive : d'une part, en observant les usages linguistiques d'un échantillon représentatif de locuteurs, observation facilitée matériellement par le fait que l'auteur est locutrice native ; d'autre part, en exploitant la compétence linguistique de locutrice native de l'enquêtrice elle-même, avec la conscience que cet avantage a des revers, nuisant justement à l'objectivité scientifique. C'est donc la combinaison de deux sources - premièrement, les résultats produits par le dépouillement des questionnaires et corpus et, deuxièmement, la compétence linguistique de la chercheuse-locutrice - qui a permis de rédiger le premier inventaire lexicographique du français calédonien. Il s'agit plus précisément de deux objets : (i) des articles lexicographiques, soit 500 pages environ réduites pour publication à 144 pages (Pauleau 1995a), et (ii) d'une analyse lexicologique de 500 pages environ, dont certains éléments ont donné lieu à publication (Pauleau 1995b). Les principes méthodologiques et théoriques de ces analyses sont ceux de la recherche descriptive différentielle, fondant l'observation d'un corpus de " particularismes " par différence avec un corpus de référence. Il s'agit de décrire ce qui diverge entre les particularismes du corpus à étudier (formes lexicales particulières du point de vue au moins diatopique) et les formes du français répertoriées par le corpus de référence : les dictionnaires tels que le Petit Robert voire le Grand Robert ou encore le Trésor de la Langue Française (TLF) - ce dernier rassemblant plus largement un « corpus d'emploi », un français global ou tendant vers le global, i.e. un large ensemble d'usages hexagonaux et extra-hexagonaux parmi lesquels se trouve le français dit de référence. D'autres ouvrages, spécifiquement consacrés à la description de lexiques non standards comme le Dictionnaire du français parlé (Bernet \& Rézeau 1989) ${ }^{9}$, font également partie des outils d'observation, permettant ainsi de confronter le lexique calédonien non seulement à la norme de référence mais aussi à d'autres lexiques non standards. Confronter un corpus de lexique polylectal calédonien à un corpus de lexique polylectal général, français de référence et autres formes de français, tel est l'objectif méthodologique, une évidence peut-être, mais que nous énonçons ici par souci de clarté. Chaque lexie retenue est décrite dans le détail de rubriques multiples de la manière suivante, les rubriques entre parenthèses étant facultatives :

9. Bernet \& Rézeau (2008) n’était malheureusement pas encore paru au moment de la rédaction des articles. 
ENTREE [prononciation] : (Attesté uniquement à l'oral) catégorie(s) grammaticale(s). Marque(s) d'usage ${ }^{10}$. (Domaine - si l'usage est spécialisé). (Identification scientifique pour les termes de la flore ou de la faune). Définition(s). (Connotation(s)). (Encycl. : rubrique encyclopédique). (Illustration(s) Référence de l'illustration, sauf si celleci a été forgée - cas rares ${ }^{11}$ ). (Équivalent hexagonal ${ }^{12}$ ). (Syn.) (Ant.) (Variante(s)). (Locution(s)). (Dérivé(s)). (Composé(s)). (Collocation(s) fréquente(s)). (Norme ${ }^{13}$ ).

(LOCUTION ou COLLOCATION : nouvel article)

(COMPOSE : nouvel article)

(ENTREE 2 : nouvel article, l'entrée initiale est alors numérotée ENTREE 1)

Du point de vue théorique, la vision globale de la francophonie adoptée par ce travail serait proche de celle présentée par R. Chaudenson (voir Chaudenson 2000, entre autres), du fait de la prise en compte de chaque lieu de la francophonie en tant que situation du français dans la francophonie et de la distinction, pour chaque situation, de deux types d'éléments : le status (institutions, fonctions, représentations) et le corpus (productions et compétences), selon les facteurs géopolitiques, historiques, sociolinguistiques.

De nouvelles enquêtes de terrain (récolte de nouveaux corpus et distribution de nouveaux questionnaires à un nouvel échantillon représentatif), menées quinze ans après celles qui ont été évoquées supra, ont permis de publier une version revue et augmentée de la description lexicographique de C. Pauleau (1995a). Ce nouvel inventaire lexicographique (Pauleau 2007) présente non seulement une nomenclature plus importante mais aussi des précisions concernant l'usage des lexies : notamment une rubrique «Dynamique » a été insérée dans les articles, dans laquelle est mentionnée l'évolution des emplois, en synchronie dynamique. Certains items passent en effet en quinze ans de vieillis à obsolètes par exemple ${ }^{14}$, d'autres apparaissent comme nouveaux, car présentés comme tels par les jeunes lycéens, et étaient effectivement absents de la récolte précédente ${ }^{15}$ (quelques exemples de ces descriptions lexicographiques sont donnés en annexe ici même). En introduction de l'ouvrage de C. Pauleau (2007), un bilan de cette dynamique lexicale pose que, selon les enquêtes de terrain, la majorité des lexies ont un usage stable : ces lexies sont toujours apparemment connues

10. Marques sociolinguistiques : « sociolectal» («pop. » ou autre), « ethnolectal» (« kanak », « polynésien » ou autre), «familier», « soutenu », « savant», « vieilli », « obsolète ». Marques quantitatives : de « très courant » à « rare ».

11. Très peu d'entrées ne sont illustrées que par des exemples d'énoncés forgés par l'auteur, le travail de terrain permettant d'éviter cela.

12. Lorsque l'entrée relève d'une variété intralinguistique ayant un équivalent en français hexagonal (p. ex. la « variété familière »), cet équivalent est mentionné (un caillou 'une belle fille' équivalent hexagonal : un canon).

13. Il s'agit dans cette rubrique de précisions sur la norme, notamment d'éléments de comparaison par rapport à la norme hexagonale décrite par les dictionnaires usuels.

14. Comme l'expression Ä̈ta pea pea 'Ne t'inquiète pas', empruntée au tahitien, qui était courante dans les années 80 mais qui semble obsolète désormais.

15. Comme l'adjectif choc 'formidable' (C'est choc ! 'C'est formidable !'), expression aujourd'hui courante mais nouvelle au moment des enquêtes (2005). 
et employées de manière courante (même si la synchronie est dynamique, avec des formes sortant de l'usage et d'autres entrant dans l'usage, comme évoqué supra). Un bilan de l'analyse polylectale et sociolinguistique montre que les innovations lexicales sont toujours nombreuses malgré la pression du modèle de la norme de référence car la standardisation par les médias existe comme ailleurs : la presse locale s'est accrue de manière spectaculaire à partir des années 2000 et elle expose les locuteurs à un français de plus en plus standardisé ${ }^{16}$. En outre, le modèle de l'anglais, dont la présence est forte dans le français des jeunes francophones en général (en Nouvelle-Calédonie comme ailleurs), vient faire écho, sur le terrain calédonien, aux nombreux emprunts anciens à l'anglais austral ${ }^{17} \mathrm{ou}$ à l'anglais américain ${ }^{18}$. Ce bilan souligne également l'importance sur le terrain calédonien de la variation diastratique ainsi que du domaine des mots dits vulgaires. En effet, parmi les sociolectes du français calédonien, le français populaire calédonien est le plus marqué par les différences par rapport à la norme de référence. Cela correspond au cas général mais, en revanche, le français populaire calédonien, contrairement au cas général, a un usage étendu aux divers groupes socio-économiques, les populations riches usant souvent du même (ou quasiment du même) français populaire que les populations pauvres ${ }^{19}$. L'usage du français populaire calédonien manifeste ainsi une culture populaire davantage qu'une classe sociale défavorisée. En outre, les « gros mots » non seulement sont nombreux mais sont emblématiques de l'identité linguistique calédonienne ${ }^{20}$.

\subsubsection{Une base de données lexicographiques en accès libre : la BDLP-Nouvelle-Calédonie}

En parallèle du travail lexicographique, évoqué au point précédent, une base de données lexicographiques a été conçue et alimentée à partir de 2005 au sein de la Base de Données Lexicographiques Panfrancophone (www.bdlp.org) qui existe depuis 1999 au sein du Trésor de la Langue Française au Québec ${ }^{21}$. Cette base de données se fonde sur des principes théoriques et méthodologiques tels que la primauté du travail de terrain, la non hiérarchisation des faits à décrire, le principe de description différentielle par rapport à un français de référence (dans le sens de " corpus de référence ", voir supra), l'objectif étant de témoigner du

16. Telle recette de cuisine mentionne par exemple dans ses ingrédients « la coriandre » et la « noix de coco » (journal gratuit, 2005) alors que les recettes écrites localement parlent d'ordinaire, comme on l'a toujours fait en Calédonie, de persil chinois (éventuellement de coriandre mais au masculin) et de coco.

17. Creek pour 'rivière' par exemple, ainsi que de nombreux termes de l'élevage. Voir note 6.

18. Wharf ('ponton') ou car port ('abri pour voiture'), voir Thibault (2016, ce volume). Voir également la présentation du présent ouvrage (Pauleau 2016b), à propos de la présence massive d'anglophones en NouvelleCalédonie lors de la Seconde Guerre mondiale.

19. Voir à ce sujet les contributions de Ledegen (2016) et Thibault (2016) dans le présent ouvrage.

20. Voir l'entrée L'enculé ! en annexe.

21. Cette base de données rassemble aujourd'hui des descriptions lexicographiques d'une vingtaine de régions francophones. 
patrimoine linguistico-culturel humain ou encore de dresser le portrait d'un « français universel » (Poirier 2005).

La source première d'alimentation de la BDLP Nouvelle-Calédonie est constituée par les ouvrages publiés sur le sujet (Pauleau 1995a, 2007). Toutefois, d'autres observations de terrain plus récentes et d'autres attestations d'occurrences postérieures à 2007 (en grande majorité non publiées) sont souvent exploitées pour nourrir les rubriques de ce site Internet. De nouvelles entrées ont également été ajoutées à la nomenclature ${ }^{22}$ et cela va se poursuivre ; cette base de données étant pour la Nouvelle-Calédonie le support désormais unique de la publication des descriptions lexicographiques ainsi que l'état le plus actualisé de la description. Mais malheureusement, ce travail d'alimentation de la base est proprement chronophage, les formats scientifiques et techniques n'étant pas les mêmes pour les descriptions papier et pour les descriptions de la base électronique : il s'agit d'un travail véritablement lexicologique et lexicographique et non pas, comme on pourrait le croire, d'une simple saisie des données. C'est ainsi que l'on ne compte à ce jour que 805 fiches dans la base calédonienne, l'alimentation de la base, travail de fourmi, avançant très lentement.

\subsection{Travaux concernant la prononciation du français calédonien}

\subsection{1. Étude contrastive instrumentale}

En 1988, une première description phonétique (Pauleau [1988] 2013) a été effectuée dans une perspective de phonétique contrastive instrumentale. Les traits emblématiques de l' « accent calédonien », notamment la confusion des nasales [õ] et [ã] ${ }^{23}$ ou l'oralisation des consonnes sourdes notamment la sifflante $[\mathrm{s}]^{24}$, ainsi que les traits moins saillants, tels que la fermeture du [c] en [e] ${ }^{25}$, la postériorisation $\mathrm{du}[\mathrm{a}]{ }^{26}$, ou encore l'oralisation $\mathrm{du} \ll \mathrm{r} »{ }^{27}$ ont été répertoriés et présentés comme constituant la prononciation commune aux divers groupes ethnolinguistiques, même s'il existe en outre des « accents » de groupe (voir le point suivant). La description est illustrée par des corpus oraux récoltés par entretien non directif (un corpus produit par un locuteur de Nouméa, un autre par un locuteur parisien), également soumis au spectrographe afin de comparer les formants des voyelles et des consonnes dans les deux corpus. En 2010, dans le cadre d'un projet PFC, la description effectuée en 1988 a été actualisée (voir point suivant).

22. Évasaner 'envoyer en évasan (évacuation sanitaire)', Kamadja 'Blanc' (les Kamadja 'les Blancs'), aire coutumière ('aire dont les frontières sont relatives à la coutume kanak'), etc.

23. «ponton » prononcé [pãtã] ; « en arrivant » prononcé [õnarivõ].

24. «C'est ça aussi » (interjection fréquente d'acquiescement polysémique) prononcé [zezaozi].

25. « fête » prononcé [fet].

26. « partir » prononcé avec un « a postérieur ».

27. En forçant à peine le trait, la prononciation des mots « leur colère », par exemple, ressemblerait à leuar golèar $(\ll \mathrm{r} »$ devient quasiment une voyelle « $\mathrm{a} »)$. 


\subsubsection{Projet PFC (Phonologie du Français Contemporain)}

Le domaine phonique est également représenté par de plus récentes recherches que celles évoquées au point précédent : une enquête de terrain a été menée en Nouvelle-Calédonie en 2010 au sein du projet PFC : Phonologie du Français Contemporain (Pauleau 2013). Cette enquête met à jour et illustre la description de la prononciation du français calédonien par de nouveaux corpus (données orales récoltées selon le protocole de $P F C$, par enregistrements de locuteurs lisant une liste de paires de mots ainsi qu'un texte, puis s'entretenant de façon guidée puis libre avec l'enquêteur). Une autre enquête de terrain du programme PFC avait été envisagée en 2006 (mais non poursuivie) par un chercheur de l'Université de la Nouvelle-Calédonie sur les « accents » des divers groupes ethnolinguistiques en présence dans l'île. Au contraire, l'étude rapportée dans C. Pauleau (2013) se focalise sur les points communs phonétiques que l'on trouve dans ce que l'on pourrait appeler l' «accent calédonien général », celui des Blancs, des Métis et de nombreux locuteurs d'autres groupes, accent que l'on appelle localement " accent calédonien », les accents plus groupaux étant appelés selon le groupe " accent kanak», " accent wallisien », " accent tahitien ", etc.

L'observation des enregistrements d'un échantillon représentatif d'une douzaine de locuteurs (tel que prévu par le protocole $P F C$ ) montre que la prononciation du français calédonien n'a pas changé par rapport à celle qui avait été décrite en 1988, mais qu'elle s'est diluée au sein d'une population beaucoup plus nombreuse et variée qu'autrefois. En particulier, l'immigration en provenance de la Métropole a rendu l'accent moins visible à Nouméa (seule ville de l'archipel). Ces analyses, instrumentales ou "à l'oreille», sont secondées par le sentiment linguistique et les observations de l'auteur-locutrice-native. Les descriptions effectuées sur le plan phonétique nécessitent aujourd'hui d'être poursuivies notamment sur le terrain de la « brousse ", i.e. les régions hors de la ville de Nouméa, et complétées par une étude phonologique.

\subsection{Autres travaux}

Divers autres travaux ont exploré des sujets variés concernant le français de Nouvelle-Calédonie. M. Darot et C. Pauleau (1993) ont brossé un tableau général de la situation du français en Nouvelle-Calédonie. C. Pauleau (2000b) a décrit le contexte calédonien en l'insérant dans la situation océanienne. C. Pauleau (1994) a aussi décrit l'imaginaire linguistique du locuteur de français calédonien et montré quelle est la valeur identitaire du parler sur ce terrain : parler le français calédonien, c'est être Calédonien. Dans le même domaine, C. Pauleau (1997) a posé la question lexicale, sociolinguistique et identitaire de l'opposition Calédonien vs. Caldoche et C. Pauleau (2008) a observé les représentations liées à l'objet "dictionnaire », qui sont complexes et variées, dans les "souscommunautés », telles que celle des locuteurs de français calédonien, comme dans la communauté globale. La méthodologie en lexicologie et en lexicographie 
différentielle a également été interrogée sur le terrain calédonien (Pauleau 1995b) pour les problèmes de classement et les questions spécifiques posées par les variantes lexicales calédoniennes mettant en jeu les plans phonique, syntaxique ou morphologique ${ }^{28}$. Autre question méthodologique ayant été traitée par le biais des recherches sur le terrain calédonien : celle de l'importance du statut de chercheur-locuteur-natif dans le travail de terrain (Pauleau 1995c), statut permettant de naviguer entre la compétence linguistique en français de référence et la compétence en français régional. Enfin, C. Pauleau (2006) a établi un bilan concernant la synchronie dynamique et son observation par le biais des enquêtes de terrain en posant la question de l'évolution des pratiques linguistiques sur une période de quinze ans. Ce thème de la dynamique, associé à celui de la place de l'idiome franco-calédonien en tant que français régional au sein d'un contexte général mondialisé, a été abordé plus récemment (Pauleau 2016a). Ajoutons qu'un ouvrage de vulgarisation a également été publié pour mieux faire connaître au grand public cet idiome des antipodes (Pauleau 2000a) ${ }^{29}$.

De leur côté, les collègues africanistes qui ont travaillé dans les années 90 au Dictionnaire Universel Francophone (DUF 1997) ont fait mention de quelques données du français calédonien. J.-M. Charpentier, linguiste spécialiste du Vanuatu, a été responsable de la base "Vanuatu, Nouvelle-Calédonie » pour le DUF, ce qui a ainsi permis à la Nouvelle-Calédonie d'être représentée dans cet ouvrage. Les données calédoniennes y sont malheureusement très peu nombreuses. Pour illustration, l'article James Cook mentionne les pays que ce navigateur a découvert mais pas la Nouvelle-Calédonie ; ou encore l'emblème du pays, un oiseau appelé cagou, est mentionné en tant qu'oiseau mais non en tant qu'emblème national calédonien ; l'article coolie mentionne les usages de ce mot du « français colonial » dans plusieurs régions mais pas la Nouvelle-Calédonie, etc. (la liste de ces absences est longue). Les collègues de Nouvelle-Zélande ont continué à évoquer un peu le français de Nouvelle-Calédonie dans des ouvrages généralistes sur le Pacifique, linguistiques (Corne \& Hollyman 1996) ou non linguistiques (Tryon 1991), en orientant leurs éléments descriptifs sur les aspects historiques de la présence du français en Nouvelle-Calédonie et sur ses particularités générales, surtout celles liées au contact des langues. Plus récemment, P. Rézeau (2008) s'est intéressé à un corpus de romans policiers contenant un certains nombre de

\footnotetext{
28. Par exemple, certaines interjections de surprise comme Babylone ! Bataillon! Baptiste ! ou Bathyscaphe! sont créées à partir de signifiants du français de référence choisis sur le critère phonique d'une première syllabe en [ba] suivie de 2 syllabes. D'autres le sont sur une première syllabe en [ka], à trois ou deux syllabes : Canasson! Calisson! Calice! Cela fait donc intervenir le plan phonique dans la néologie lexicale. Pour d'autres lexies, c'est le plan syntaxique qui est en question, par exemple le verbe parler mauvais ('médire') est un fait de néologie lexicale se caractérisant par un fait syntaxique, la dissociation de morphèmes.
}

29. Cet ouvrage de vulgarisation est le seul livre sur le français calédonien a avoir été choisi pour une mise en vente à la librairie du Musée Branly lors de l'exposition Kanak, l'art est une parole en 2014, ce qui montre l'utilité de ce type d'ouvrage. 
calédonianismes ${ }^{30}$ : le chercheur a dépouillé ce corpus et constitué un excellent glossaire (105 pages) aux articles très richement documentés ${ }^{31}$.

\section{CONCLUSION}

Faire le bilan des résultats de recherche sur le français calédonien et les rassembler ici, tel a été notre objectif, permettant ainsi de produire un outil de travail inédit. Des étapes initiales néo-zélandaises fondatrices de toute la dynamique d'investigation sur le terrain francophone calédonien à partir des années 70, aux recherches ultérieures et actuelles menées dans des universités françaises, les travaux sont en réalité peu nombreux et portent sur des domaines restreints. Celui du lexique est très fortement majoritaire, surtout par la description lexicographique sous forme d'inventaires différentiels, l'aspect phonétique étant également quelque peu observé, notamment par une enquête du programme Phonologie du Français Contemporain. Les publications sur le français calédonien qui portent au public les recherches ainsi évoquées sont majoritairement locales, pour des raisons tant matérielles (les éditeurs de Nouméa étant en demande d'ouvrages de ce type) que politiques et culturelles : il était important, pour participer à la construction identitaire de ce pays en plein processus d'autodétermination ${ }^{32}$, de publier localement ces ouvrages.

Les recherches évoquées dans cet article, publiées localement (ou nationalement pour certaines), valident et confirment l'hypothèse de l'existence et de la densité du français calédonien et l'état des lieux effectué ici devrait permettre, notamment aux jeunes chercheurs, de prendre la mesure du travail qui reste à faire sur ce terrain pour explorer cette variété de langue :

- dans le domaine du lexique, l'aspect étymologique doit être étudié, l'origine de chaque lexie n'ayant $\mathrm{pu}$, en cet état de la recherche lexicographique, être étudiée systématiquement. La contribution d'I. Wissner (2016, ce volume) montre d'ailleurs explicitement le chemin pour de telles futures recherches, notamment dans une perspective panfrancophone, permettant de confronter les formes du français prises en Nouvelle-Calédonie et ailleurs dans la francophonie ;

- sur le plan phonique, l'aspect phonologique doit être traité puisque les seuls travaux existants se sont intéressés à la variation phonétique uniquement ;

- la syntaxe en soi constitue également un pan entier non observé de façon soutenue puisque, jusqu'au présent ouvrage, seules quelques remarques ont

30. Romans d'A.D.G., un auteur métropolitain ayant vécu à Nouméa.

31. Rézeau présente la Nouvelle-Calédonie comme «l'une des aires francophones dont le lexique a sans doute été le mieux étudié [par les travaux] remarquables et complémentaires de K. James Hollyman et de Christine Pauleau »)... mais « l'une des moins bien représentées dans les dictionnaires généraux du français » (2008: 454).

32. Voir l'introduction du présent ouvrage (Pauleau 2016b), notamment la première annexe. 
été développées au sein de la description lexicale, concernant les créations de néologie lexicale mettant en jeu la syntaxe (voir supra §3.3). Le texte de D. Bottineau donne dans les pages qui suivent certaines pistes dans ce domaine ;

- d'une façon plus générale, d'autres champs pourraient être explorés comme celui des pratiques langagières, qui pourrait faire l'objet d'une étude dynamique (à envisager par exemple dans l'ensemble du répertoire linguistique calédonien) ;

- un dernier exemple s'impose, celui des études conversationnelles mettant en relation le verbal et le non-verbal, prenant en compte des faits tels que la gestuelle et les expressions du visage (les Calédoniens savent tous, par exemple, qu'ils n'ont pas besoin de leurs cordes vocales pour acquiescer le propos d'un interlocuteur, car hausser les sourcils suffit pour dire « oui » en Nouvelle-Calédonie).

Autant de thèmes à explorer de façon pionnière sur ce terrain lointain, méconnu, caractérisé par le métissage culturel de $l^{\prime}$ " occident » et de $l^{\prime}$ " orient », et qui se trouve être un solide représentant de la francophonie en Océanie (voir l'introduction du présent ouvrage).

\section{ANNEXES}

\section{Exemples lexicographiques (in Pauleau, 2007 : 117)}

LANTERNE : n.f. Cour. (Priacanthus hamrur(Forssk.) - Observatoire, 1989 : 131). Poisson rouge à gros yeux dont la chair est "délicate et fondante" (ibid.). Ah oui les lanternes c'est les poissons à gros yeux là... Oral d'enquête, 1991.

LA POP : Attesté uniquement à l'oral. loc. adj. Cour. Fam. ou sociolectal (pop.) et/ou ethnolectal kanak*. Vieilli. A la mode. C'est un mec la pop, lui... Oral spontané, 1990. Syn. : V. KAYA FOU.

LAVER LA GUEULE + complément : Attesté uniquement à l'oral. loc. verbale. tr. indir. Cour. Fam. ou sociolectal (pop.). Parfois plaisant. Frapper + complément. V. DECALQUER LA GUEULE. Dis lui qu'il arrête sinon j'vais lui laver la gueule! Collocation fréquente : Laver la gueule à coups de baffes.

LELEÏPE ? / LELEÏPE ! : Attesté uniquement à l'oral. phrase (affirmative ou interrogative). T.cour. Fam. ou sociolectal pop. Ethnolectal polynésien/kanak* (peu cour. chez les Européens). Ça va ? / Ça va ! V. BOULETTE [Deux personnes qui se rencontrent :] - Leleïpe ? - Leleïpe! Dynamique : Selon l'enquête de 2005, terme toujours très en usage, et toujours nettement sociolectal (pop.).

L'EMPÉTÉ ! : Attesté uniquement à l'oral. interj. T.cour. Fam. Vulg. Exprime la surprise. V. L'ENCULÉ ! et BABYLONE ! Hé !* L'empété ! Mais arrête de manger dans mon assiette! Norme : Le degré de vulgarité est bien moindre qu'il ne serait 
en français hexagonal pour la même interjection (registre vulgaire banalisé en contexte calédonien*). Dynamique : Selon l'enquête de 2005, terme stable.

L'EN ! : Variante atténuée de L'ENCULÉ ! V. cette entrée. [en voiture :] L'en ! $T^{\prime}$ as vu tous les gendarmes ? Norme phonétique : Se prononce avec allongement vocalique : [lãã]. Dynamique : Selon l'enquête de 2005, terme stable.

L'ENCULÉ ! : interj. T.cour. Fam. ou sociolectal (pop.). Registre vulg. souvent neutralisé. Marque l'intensité d'une réaction, qu'elle soit de surprise, de colère, de dégoût, d'amusement, de désapprobation, d'admiration, etc. et possède également une fonction de démarcatif (ponctue le discours en délimitant les groupes de sens - V. dernière illustration). Encycl. : Avec quelques autres lexies (notamment des interjections comme L'engin !* ou Valable !*) L'enculé ! fait partie des stéréotypes lexicaux calédoniens*, notamment lorsqu'il s'agit du Calédonien* d'origine européenne ou, du moins, de sa caricature, ancrée dans la conscience collective (du groupe européen* surtout) comme stéréotype drôle et marginal par rapport à la norme hexagonale. Les humoristes locaux en font grand usage dans leurs sketches. Après trois séjours, il [le métropolitain] a bien changé : quand il y a un problème il dit : "L'enculé !" Sketch Ollivaud, 198? L'enculé ! Jacques ! Mon frère! Sketch Lewis, 1990. Enfin on a quand même fait une bonne pêche (.) et puis l'enculé ! des pièces* hein ? Sketch Valéry, 1989. - Ben l'enculé ! Vous avez pas peur vous ! Roman Tcherko, 2001 : 29. Longulé. (.) Longlé ! On a oublié (.) BD Teg, 2002 : 1418. Hulé ! sont tombés par terre! [les nautous* prêts à cuire]. Recettes Moglia, 2004 : 72. L'enculé ! Mais t'as vu cet abruti d'chien ? L'enculé ! Il a même pas aboyé, c'est un nul! Ah* l'enculé ! Équivalents hexagonaux (ils sont nombreux) : Mon vieux ! (français fam. traditionnel). Putain ! (français fam. des jeunes). Putain con!, Boudu! (français régional du sud ouest). Purée! (français pop.), etc. (le rôle de démarcatif étant le même). Syn. (ils sont nombreux) : V. BABYLONE ! Variantes atténuées (degré de vulgarité atténué) : L'en !* L'engine !* (attestées uniquement à l'oral). Culotte !* L'empété !* L'enculotte !* L'engin !* L'ôngin !*. Collocations fréquentes : V. articles ci-après. Norme : Le degré de vulgarité est bien moindre qu'il ne serait en français hexagonal pour la même interjection (registre vulgaire neutralisé en contexte calédonien*). Notons tout de même que, dans certains milieux hexagonaux populaires ou régionaux, on observe également ce phénomène de neutralisation de la vulgarité : en français méridional familier, par exemple, l'usage de l'interjection "Enculé !" étant courant, le registre vulgaire est ainsi neutralisé comme en contexte calédonien*. / Le terme est attesté uniquement à l'oral selon les enquêtes de 1995 mais attesté à l'écrit à partir de 2001 : cela manifeste peut-être une banalisation croissante du registre vulgaire de cette expression et la levée des censures de l'écrit en contexte local. Norme phonétique : Se prononce parfois avec allongements vocaliques, surtout de la première et la troisième syllabe (allongements proportionnels à l'intensité de la réaction exprimée) : [lããkyle], [lãkylee] ou [lããkylee]. Souvent prononcé aussi sans la première syllabe : [kyle] (ou [kølø] orthographié "keuleu" par un informateur lycéen en 2005), ou même [yle] "Hulé !", comme le montre l'exemple de Moglia ci-dessus. La prononciation illustrée par Teg ci-dessus correspond 
encore à une autre prononciation du français calédonien* populaire (avec la voyelle nasale fermée et la consonne [k] voisée) : "longulé" [lõgyle] ou "longlé" [lõgle]. Toutes ces manières de prononcer correspondent aux diverses variations en usage. Dynamique : Selon l'enquête de 2005, terme stable.

\title{
2. Datations et témoignages d'enquête (in CNRS-INALF, 1996 : 94)
}

\author{
DATATIONS ET TÉMOIGNAGES D' ENQUÊTE
}

Matérfaux calédoniens préparés par K.J. Hollynan

Enqueteurs
AB A. Butler Jh K. J. Hollynan
CC C. Corne LO LIse Ourgaud
JG J. Glasgow

\footnotetext{
BOIS BALAI sn (bot) Herbe à balat (v. OFPED 3,91,199b) adulte, devenue 11gneuse. (Enq JH 1966: Th10).

BOIS NAVET sm (bot) Pisonia aculeata L. (Nyctaginocés), (Enq JH 1966).

BOUC sn (adniratif) home/garçon fort (na1s peu intelligent) (Enq JH 1961); (péjoratif) vieux bouc vieux coureur de fupons (Enq AB 1962); petit bouc, petit 1diot (Enq AB 1962).
}

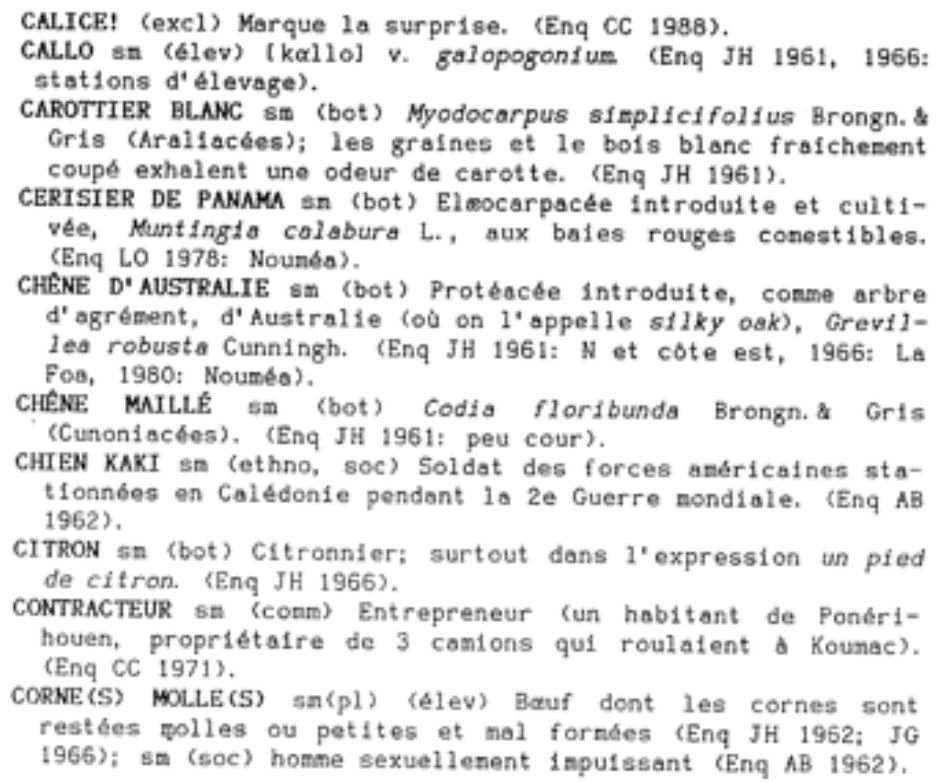




\section{Références}

[BDLP] Base de données lexicographiques panfrancophones, 2001-2014, AUF-TLFQ. [www.bdlp. org]

[BDLP-NouveLLE-CALÉDONIE] Base de données lexicographiques panfrancophones, volet " NouvelleCalédonie ", Christine Pauleau (dir.), 2006-2014. [www.bdlp.org/accueil.asp?base=NC]

[DUF] Dictionnaire universel francophone, 1997, Paris : Hachette.

[IRD] Bonvallot J., Gay J.-C. \& HaBert E. (éds) (2012), Atlas de la Nouvelle-Calédonie, Marseille : Institut de Recherche pour le Développement.

[PFC] Phonologie du Français Contemporain [http://www.projet-pfc.net/]

BARNÈCHE S. (2005), Gens de Nouméa, gens des îles, gens d'ailleurs... Langues et identités en Nouvelle-Calédonie, Paris : L'Harmattan.

BeRnet C. \& RÉzeAu P. (1989), Dictionnaire du français parlé. Le monde des expressions familières, Paris : Seuil.

Bernet C. \& RézeAu P. (2008), "On va le dire comme ça ". Dictionnaire des expressions quotidiennes, Paris : Balland.

Berrendonner A., Le Guern M. \& Puech G. (1983), Principes de grammaire polylectale, Lyon : Presses Universitaires de Lyon.

BotTineAu D. (2016b), "Les particularités du français calédonien (lexique, morphosyntaxe) et leurs enjeux sémantiques, pragmatiques et cognitifs ", Langages 203. (ce volume)

CHAUdENSON R. (2000), Mondialisation : la langue française a-t-elle encore un avenir ?, Paris : Institut de la Francophonie \& Didier Érudition.

CNRS-INALF (1983-1996), Observatoire du français dans le Pacifique, Paris : Didier-Érudition.

CORNE C. (1996), "La formation de deux nouvelles langues vernaculaires en Nouvelle-Calédonie, le français calédonien et le tayo ", Observatoire du Français dans le Pacifique : études et documents 11-12, 99-118.

CORNe C. \& Hollyman K.-J. (1996), "French in the south Pacific", in S. A. Wurm, P. Mühlhäusler \& D. T. Tryon (eds), Atlas of Languages of Intercultural Communication in the Pacific, Asia and the Americas, Berlin/New York: Mouton de Gruyter.

Darot M. \& Pauleau-Delautre C. (1993), "Situation du français en Nouvelle-Calédonie ", in D. de Robillard \& M. Beniamino (éds), Le Français dans l'espace francophone, Paris : Champion, 283-301.

EHRHART S. (1993), Le Tayo, un créole à base lexicale française en Nouvelle-Calédonie, Hamburg : Ed. Buske.

EHRHART S. (2012), L'Écologie des langues de contact. Le tayo, créole de Nouvelle-Calédonie, Paris : L'Harmattan.

EHRHART S. (2016), "Entre français calédonien et langue kanak : quelle place pour le tayo ? Une approche écolinguistique ", Langages 203. (ce volume)

FÉdération des CEuvres Laïques (F.O.L.) (éd.) (1983), Mille et un mots calédoniens, Nouméa : F.O.L.

GLAsgow J. (1968), Le Vocabulaire de l'élevage en Nouvelle-Calédonie : étude de français régional, Thèse de doctorat de I'Université d'Auckland, Nouvelle-Zélande.

LEDEGEN G. (2016), "Traits syntaxiques < populaires> dans le français < régional> de La Réunion : très populaires dans la francophonie, jusqu'en Nouvelle-Calédonie ", Langages 203. (ce volume) 
PAULEAU C. ([1988] 2013), Étude phonétique contrastive du français calédonien et du français standard, Paris: Maison de la Nouvelle-Calédonie. [publication numérisée, mémoire de Maîtrise, 1988]

PAULEAU C. (1994), "Le français calédonien : une valeur identitaire - un aspect sociolinguistique de l'identité calédonienne ", in Ouvrage collectif, Être Caldoche aujourd'hui, Nouméa : Île de Lumière, 179-187.

PAULEAu C. (1995a), Le Français de Nouvelle-Calédonie - Contribution à un inventaire des particularités lexicales, Paris : EDICEF-Association des Universités Partiellement ou Entièrement d'Expression Française [AUPELF].

PAULEAU C. (1995b), "La variation du français en Nouvelle-Calédonie : problèmes de classement et questions spécifiques posées par les variantes phoniques et syntaxiques ", Actes des $I^{\text {es }}$ Journées scientifiques du réseau 'Langues et français en francophonie' de l'Université des Réseaux d'Expression Française [UREF], 22-25 janvier 1994, Paris : AUPELF, 203-213.

PAULEAU C. (1995c), "L'analyse différentielle menée par un chercheur-locuteur : d'une méthodologie d'analyse du français en francophonie ", La Linguistique 31 (1), 105-117.

Pauleau C. (1997), "Calédonien et Caldoche ", Mots. Les langages du politique 53, 48-54.

Pauleau C. (2000a), Le Calédonien de poche, Paris: Assimil.

Pauleau C. (2000b), "Le français en Océanie ", in G. Antoine \& B. Cerquiglini (éds), Histoire de la langue française 1945-2000, Paris : Éditions du CNRS, 839-850.

Pauleau C. (2006), "Le français de Nouvelle-Calédonie, approche d'une synchronie dynamique (1990-2005) ", Le Français en Afrique 21, 169-176.

PAuleau C. (2007), Mots de Nouvelle-Calédonie, éléments de recherche sociolinguistique sur le français calédonien : inventaire lexicographique polylectal, Nouméa: Centre de Documentation Pédagogique de Nouvelle-Calédonie (2 tomes).

Pauleau C. (2008), "L'objet 'dictionnaire du français calédonien' : À propos de Mots de Nouvelle-Calédonie... ", in C. Bavoux (éd.), Le Français des dictionnaires, l'autre versant de la lexicographie française, Bruxelles : De Boeck-Duculot, 163-174.

PAULEAU C. (2013), "Description et sauvegarde du patrimoine immatériel de la langue francocalédonienne ", Bulletin de la Société d’Études Historiques de Nouvelle-Calédonie 175, 52-68.

Pauleau C. (2016a), " Le lexique du français calédonien. Présentation linguistique et sociolinguistique. Un <français régional> comme manifestation de la mondialisation ", in M.-M. Bertucci (éd.), Les français régionaux dans l'espace francophone, Bruxelles : Peter Lang, 141-154.

PAULEAU C. (2016b), "Le français calédonien (Nouvelle-Calédonie). Description d'un français régional, confrontations de terrains panfrancophones ", Langages 203. (ce volume)

PoIRIER C. (2005), "La dynamique du français à travers l'espace francophone, à la lumière de la Base de Données Lexicographiques Panfrancophone ", Revue de linguistique romane 69, 483-516.

RÉzEAU P. (2008), "Le français de Nouvelle-Calédonie dans les romans policiers d'A.D.G. ", in A. Thibault (éd.), Richesses du français et géographie linguistique, vol. 2, Bruxelles : De Boeck/Duculot, 453-586.

ThiBAUlt A. (2016), " Diastratismes et réallocation des variantes : français d'Amérique et de Nouvelle-Calédonie ", Langages 203. (ce volume)

TRYON D. (1991), “The French Language in the Pacific”, The Journal of Pacific History 26 (2), 273-287.

WisSneR I. (2016b), "Le français des Isles: des Antilles à la Nouvelle-Calédonie ", Langages 203. (ce volume) 